\title{
Metastatic Malignant Melanoma Presenting as Small Bowel Obstruction: A Report of a Case
}

\author{
Umobong EO ${ }^{1}$, Ojo $\mathrm{BA}^{2, *}$, Aghahowa $\mathrm{ME}^{3}$, Etim $\mathrm{O}^{3}$, Ngbea $\mathrm{J}^{2}$, Ugwu $\mathrm{VI}^{4}$, Eke $\mathrm{BA}^{5}$, Nyaga $\mathrm{T}^{6}$, DUDUYEMI BM ${ }^{7}$, \\ Jumbo GTA ${ }^{8}$ \\ ${ }^{1}$ Consultant Histopathologist, Department of Laboratory Medicine, State House Medical Centre, Abuja, Nigeria \\ ${ }^{2}$ Senior Lecturer/Consultant Anatomic Pathologist, Department of Anatomical Pathology, College of Health-Sciences, Benue State \\ University, Makurdi, Nigeria \\ ${ }^{3}$ Consultant Surgeon, Department of Surgery, Asokoro District Hospital, Abuja, Nigeria \\ ${ }^{4}$ Consultant Histopathologist, Department of Histopathology, National Orthopedic Hospital Enugu, Nigeria \\ ${ }^{5}$ Consultant Surgeon/Senior Lecturer, Department of Surgery, College of Health-Sciences, Benue State University, Makurdi, Nigeria \\ ${ }^{6}$ Resident, Department of Histopathology, Benue State University Teaching Hospital, Makurdi, Nigeria \\ ${ }^{7}$ Lecturer/Consultant Anatomic Pathologist, Department of Pathology, Kwane Nkrumah University of Science and Technology, \\ Kumasi, Ghana \\ ${ }^{8}$ Department of Medical Microbiology, College of Health Sciences, Benue State University, Makurdi, Nigeria \\ *Corresponding author: babarinde087@gmail.com
}

Received July 21, 2014; Revised August 01, 2014; Accepted August 10, 2014

\begin{abstract}
Small intestinal metastasis of any malignancy is rare. Cutaneous or ocular malignant melanomas are the most common tumors that metastasize to gastrointestinal tract. Patient often present several years after the initial evaluation and treatment for a previous cutaneous malignant melanoma with intestinal obstruction symptoms. Rarely, small bowel melanomas occur with clinical picture of acute abdomen due to perforation. We present a 68 year old female patient with a 4 month history of recurrent colicky abdominal pain, distention, vomiting associated with weight loss and passage of small pellet stool. Imaging studies using abdominal ultrasound and CT scan revealed a large lobulated hypo echoic mass between the gastric antrum and pancreas. She had an exploratory laparatomy for bowel obstruction. Surgical pathology of the excised mass with resected obstructed jejunum revealed a metastatic malignant melanoma. Reported cases of metastatic small bowel melanoma are becoming increasingly common and should be considered in patients with a previous history of primary cutaneous malignant melanoma.
\end{abstract}

Keywords: malignant melanoma, metastasis, obstruction

Cite This Article: Umobong EO, Ojo BA, Aghahowa ME, Etim O, Ngbea J, Ugwu VI, Eke BA, Nyaga T, DUDUYEMI BM, and Jumbo GTA, "Metastatic Malignant Melanoma Presenting as Small Bowel Obstruction: A Report of a Case." American Journal of Clinical Medicine Research, vol. 2, no. 4 (2014): 72-74. doi: 10.12691/ajcmr-2-4-2.

\section{Introduction}

Malignant melanoma (MM) is a relatively common neoplasm that not long ago was considered almost uniformly deadly with the great preponderant arising in the skin [1]. Reported cases of small bowel melanoma are becoming increasingly more common and this is consistent with the understanding that melanoma is the most common extra-abdominal source of small bowel metastasis [2]. Reported incidences ranging from 35\%$50 \%$ have been documented for metastatic spread of melanoma to the gastrointestinal-tract [3]. Distant metastases may also be found in the liver, lungs, bone and central nervous system including within other tumors $[4,5,6]$ and the heart $[6]$.

Symptoms of metastasis of MM to the small intestine include chronic abdominal pain with features of intestinal obstruction [7]. MM is the most common cancer that metastasize to the GI trait with frequent involvement of the small intestine $[7,8,9,10]$. Chemokine receptor CCR9 and its ligand CCL25 have been implicated as signals that allow malignant melanoma to preferentially metastasize to the small intestine [11]. Imaging modalities used to detect these small bowel lesions include computed tomography(CT) scans and upper gastrointestinal series with small bowel follow -through. Given the low sensitivity of these older generations of imaging modalities, newer helical CT scanners, 18F-2 fluoro-2deoxy-D-glucose-positron emission tomography (FDG $\mathrm{PET} / \mathrm{CT}$ and capsule endoscopy are now being recommended [11].

It is important for the surgeon to recognize metastatic small bowel melanoma as a cause of intestinal obstruction, especially in patients with a previous history of cutaneous MM [12].

\section{A Case Report}

A 68 year old retired civil servant presented with a history of recurrent colicky abdominal pain, distension, 
vomiting, weight loss and passage of small pellet stools of 4 months duration. The pain was located at the epigastrium and became progressively worse in the last few days prior to presentation. The vomiting was bilious and non-projectile. There was no associated fever, melena, hematemesis or other systemic symptoms. Five years ago, she had an excision of a melanoma involving her right fourth finger. She has had a previous exploratory laparotomy about 10 months before presentation for acute intestinal obstruction due to proximal jejuno-jejunal intussusceptions where small bowel resection and end to end anastomosis was done. Biopsy showed hemorrhagic necrosis of resected bowel. Examination showed a middle aged female in painful distress with normal vital signs. The abdomen, however, was asymmetrical with features of incisional hernia, epigastric tenderness and a slightly mobile, hard and tender epigastric mass of about $10 \mathrm{~cm}$ in diameter. An initial assessment of recurrent intestinal obstruction and soft tissue sarcoma was made. Preliminary investigation with full blood count (FBC), blood sugar profile, urea, electrolytes and creatinine (U, E \& Cr), urinalysis, chest X-ray and barium enema were normal. Imaging studies using abdominal ultrasound and CT scan revealed a large lobulated hypo echoic mass between the gastric antrum and pancreas. Patient had an exploratory laparatomy with excision of the mass and resection of obstructed jejunum. Surgical pathology of the mass and resected jejunum show infiltration of the intestinal tissue by malignant cells with prominent melanin pigmentation, marked cytologicatypia and abundant mitotic figure (Figure 1A). Special studies with S-100 protein (Figure 1B), Vimentin (Figure 1C) and Dermin (Figure 1D) confirmed metastatic MM. Patient died shortly after surgery.

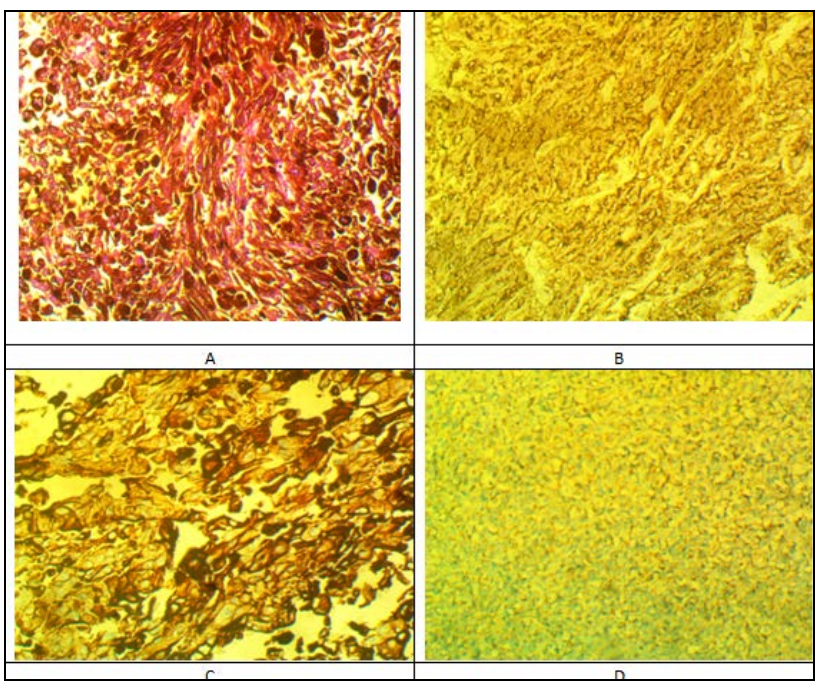

Figure 1. A composite panel of four pictures showing the characteristics histological features of malignant melanoma in this case: A) a high power magnification of the lesion shows infiltration of intestinal tissue by malignant cells with prominent melanin pigmentation (H\&E X100); B) the lesion shows strong nuclear and cytoplasmic reactivity for S-100 protein. (S-proteinX100); C) Vimentinimmunoreactivity in melanoma (VimentinX100); D) Desmin non reactivity to melanoma (DesminX100)

\section{Discussion}

Malignant melanoma is primarily a cutaneous disorder with the ability to metastasis, either locally to neighboring areas of subcutaneous tissues or also local lymph-nodes [6]. Distance metastasis has been reported especially to the GI tract, liver, central nervous system and bone $[3,4,5,6]$.

With regard to gastro intestinal metastasis, small bowel metastasis is by far most common and MM is responsible for the most frequent cause of gastro intestinal metastasis [13]. Though it appears that small bowel metastasis of melanoma is quite common, it is only of recent that the number of case reports in the literature increased significantly to mirror this observation. [13,14].

Our patient presented approximately five years after the diagnosis of a cutaneous malignant melanoma. There still exits a broad interval between diagnosis of primary malignant melanoma and identification of gastro internal metastasis anywhere between 2 to 180 months. [15].

These patients tend to present with nausea and vomiting, abdominal pain, weight loss or present with intestinal obstruction or bleeding. [14]. This patient presented with colicky abdominal pain, distention, vomiting and weight loss.

Diagnosis of metastatic malignant melanoma was established at surgical pathology. Further staining modality done to confirm the diagnosis include S-100, Dermin and Vimentin. Immunohistochemically, the typical melanoma is reactive for Vimentin, S-100 protein, HMB-45, Melan- A and tyrosinase [16,17,18] and negative for Dermin. Of these, vimentin is the most consistent with HMB-45 being more specific marker [19]. We could not stain for HMB-45 in our centre.

Surgical intervention of the intestinal obstruction via laparatomy is the standard management [20,21]. The patient had exploratory laparatomy done with excision of the mass and resection of obstructed jejunum. Metastatic melanoma has a very poor-prognosis. The averages mean survival being 6 to 8 months [22]. Most patients usually have widespread metastasis at presentation coupled with the fact that small bowel metastasis are usually clinically silent with acute presentation due to intestinal obstruction or intussusceptions being rare. [7,14,20]. Patient died shortly after operation. No autopsy was done, the possibility of other metastasis could not be ruled out in this patient.

\section{Conclusion}

Small bowel metastatic malignant melanoma is increasingly being reported. We present here a case of intestinal obstruction secondary to metastatic malignant melanoma. The patient was previously treated for cutaneous malignant melanoma approximately 5 years prior to this presentation. Imaging studies using abdominal ultrasound and CT scan revealed a mass between the gastric antrum and pancreas and its surgical pathology was consistent with metastatic malignant melanoma of the intestine.

A suspicious of metastatic malignant melanoma to intestine should always be considered as a differential diagnosis of intestinal obstruction in patient with previous history of cutaneous MM. The emphasis in the individual should be to further investigate their symptoms through imaging with subsequent laparotomy or laparoscopy. Surgical resection which has shown increase overall 
survival seems to be of little effect on our patient has she died shortly after operation. The possibility of other metastasis should be considered for this poor prognosis.

\section{Conflict of Interest}

None.

\section{Acknowledgement}

We are grateful to the Department of Surgery, Asokoro District Hospital, Abuja, Nigeria and Department of Laboratory Medicine, State House Medical Centre, Abuja, Nigeria for allowing access to their patient and data.

\section{References}

[1] Kumar V, Abbas Ak. Fausto N. The skin. Pathology Basis of Disease $7^{\text {th }}$ ed. Sauders: Elservier: 2005 p. 1234.

[2] Butte JM, Meneses M, Waugh E, Parada H, De La Fuente Hernán: Ileal intussusception secondary to small bowel metastases from melanoma. The American Journal of Surgery; 2009; 198: e1-e2.

[3] Mucci T, Long W, Witkiewicz A, Mastrangelo MJ, Rosato EL, Berger AC: Metastatic Melanoma Causing Jejunal Intussusception. Journal of Gastrointestinal Surgery; 2007; 11: 1755-1757.

[4] Adair C, Ro JY, Sabin AA, EL. Nagar AK, Ordonez NG, Ayala AG. Malignant melanoma metastatic to gastrointestinal tract. A clinicopathologic study. Int J Surgpathol 1994. 2: 3-10.

[5] Di Tommaso L, Rahal D, Bresciani G, Ronacalli M. Cutaneous melanoma metastatic to uterine asenomoyoma 223-225.

[6] Gibbs P. Cebon J.S. Calafiore P. Robinson WA. Cardiac metastases from malignant melanoma. Cancer 1999, 85: 78-84.

[7] Dogan M. Ozdemir, S, Gecim E. Intestinal malignant melanoma presenting with small bowel invagination: a case report. Turk. J Gastroenterol 2010; 21 (4): 439-42.

[8] Tarcoveanu, E, Dimofte G, Danila N, Vasilescu A, Ferariu D, Crumpei F. Small bowel malignant melanoma-report of three cases and review of literature. Acute ChirBelg 2009; 109(6):763-8.
[9] Atmatzidis KS, Pavlidis TE, Papaziogas BT, Papziogas TB. Primary malignant melanoma of the small intestine: report of a case. Surg. Today 2002; 32 (9): 831-33.

[10] Guranya SY, AI Naami M, AI Tuwaijir T, Rafah MMI.Malignant melanoma of small bowel with unknown primary. A case report. J Ayub. Med. Coll Abbottabad 2007; 1991): 63-5.

[11] ParkJ, OstrowitzMB, CohenMS, Al-Kasspooles M.A patient with metastatic melanoma of the small bowel. Oncology. 2009; Jan: 23 (1); 98-102.

[12] Lianos GD, Messinis T, Doumos R, Papoudou-Bai, Bali CD. A patient presenting with acute abdomen due to metastatic small bowel melanoma: a case report. Journal of Medical Case Reports 2013; 7: 216.

[13] P. Florus, B. Rar. Small bowel intussuception due to malignant melanoma of unknown primary with adrenal surgery at presentation. The Internet Journ. of Surg. 2010 vol. 26 (2).

[14] Patti R, Cacciatori M, Guercio G, Territo V, Di Vita: Intestinal melanoma: abroad spectrum of clinical presentation. Int J Surg Case Rep 2012, 3 (8): 395-398.

[15] HuangYJ,WuMH,Lin MT: Multiple small bowel intussusceptions caused by metastatic malignant melanoma.The American Journal of Surgery; 2008; 196: e1-e2.

[16] De Wit NS, Van Muijen GM, Ruiter DS. Immunohistochemistry in melanocytic proliferative lesions. Histopathology 2004, 44:517541.

[17] Mangini J. Li N, Bhawan J. Immunohistochemical markers of melanocytic lesions: a review of their diagnostic usefulness. Am. J. Dermatopol 2002. 24: 270-281.

[18] Yazyi H. Gown AM. Immunohistochemical markers of melanocytic tumors. Int. J SurgPathol 2003, 11: 11-15.

[19] Bacchi CE, Boneth F, Pea M, Martignomi G. Gown AM HMB-45: a review. Appl. Immunohistochem. 1996. 4:73-89.

[20] Hao SH, Li Q, Chen H. small bowel metastasis of melanoma: Palliative effect of surgical resection Jpn J Clin Oncol 1999; 29 (9): 442-44.

[21] Wong LS, Boughil I, Odugwu S, Roberts PN. Metastatic melanoma of the small bowel as a cause of acute intestinal bleeding. JR Coll Surg Edinb. 1999; 44: 241-3.

[22] Gatsoulis N, Roukounakis, N, Kafetzi I, Gasteratos, Mavrakis G: Small bowel intussusceptions due to metastatic malignant melanoma. A case report. Techniques on Coloprotology, 2004; 8: S141-S143. 